Ophthalmologe 2018 $\cdot 115: 613-614$ https://doi.org/10.1007/s00347-018-0731-z Online publiziert: 8. Mai 2018

(c) Springer Medizin Verlag $\mathrm{GmbH}$, ein Teil von Springer Nature 2018

CrossMark

Berufsverband der Augenärzte Deutschlands e.V. (BVA) $\cdot$ Deutsche Ophthalmologische Gesellschaft (DOG) ${ }^{2} \cdot$ Retinologische Gesellschaft (RG) e.V. ${ }^{3}$

'Berufsverband der Augenärzte Deutschlands e. V., Düsseldorf, Deutschland

${ }^{2}$ Deutsche Ophthalmologische Gesellschaft, München, Deutschland

${ }^{3}$ Retinologische Gesellschaft e.V., Freiburg, Deutschland

\title{
Erratum zu: Stellungnahme des Berufsverbandes der Augenärzte Deutschlands, der Deutschen Ophthalmologischen Gesellschaft und der Retinologischen Gesellschaft zur Chorioretinopathia centralis serosa (CCS). Stand Januar 2018
}

Erratum zu:

Ophthalmologe 2018

https://doi.org/10.1007/s00347-018-

0680-6

Sehr geehrter Leser, sehr geehrte Leserin,

die Onlineversion dieses Artikels wurde geändert: Leider wurde der Beitrag mit fehlerhafter • Abb. 1 veröffentlicht, auch hat sich im Abschnitt Kernaussagen/ Therapie/Lasertherapie ein Fehler eingeschlichen. Zusätzlich wurde der Berufsverband der Augenärzte Deutschlands (BVA) falsch abgekürzt.

Wir bitten darum, den korrekten Absatz sowie die neue Abb. $1 \mathrm{zu}$ berücksichtigen und den Fehler zu entschuldigen.

Die Redaktion

\section{Kernaussagen}

Therapie

- Lasertherapie: Extrafoveale Quellpunkte können mit einer Laserkoagulation therapiert werden. Zentrale Quellpunkte können mit einer unterschwelligen Lasertherapie therapiert werden.

\section{Korrespondenzadresse}

Deutsche Ophthalmologische Gesellschaft (DOG)

Deutsche Ophthalmologische Gesellschaft Platenstr. 1, 80336 München, Deutschland geschaeftsstelle@dog.org 


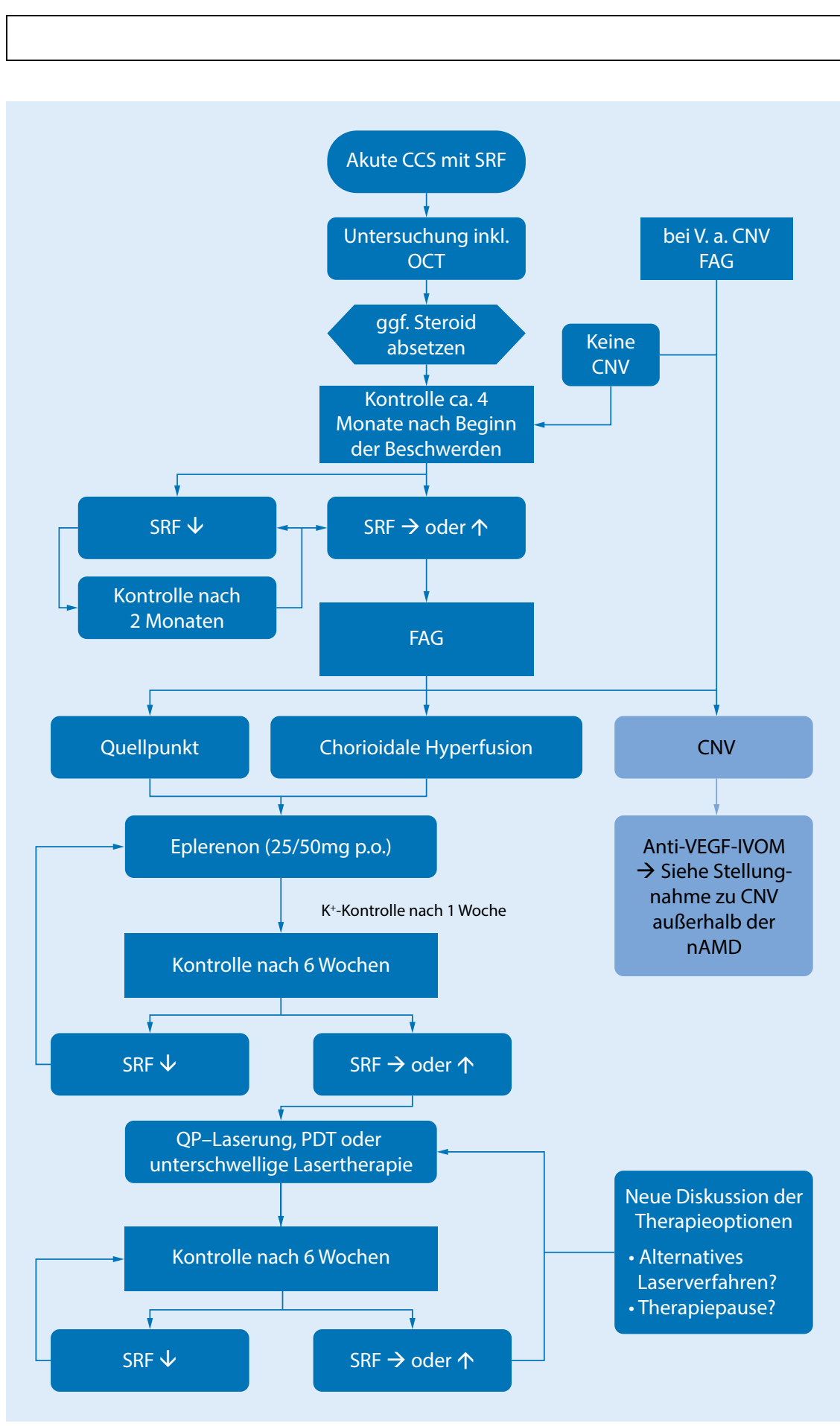

Fachnachrichten

\section{Wearables und Apps - Lücken im Gesundheitswissen}

\section{Erstaunlicherweise verfügen Träger von Wearables - obwohl sie Daten und Informationen als Grundlage für ihr Gesundheitsverhalten nutzen - nicht generell über mehr Gesund- heitswissen. Das ergab eine aktuelle empirische Studie.}

Fitnessarmbänder und Gesundheits-Apps werden von etwa jedem dritten Bürger genutzt - Tendenz steigend. In einer Studie der Hochschule Fresenius in Köln wurde nun untersucht, ob Wearables nur ein Mode-Gag sind oder vielmehr Ausdruck eines sich verändernden Umgangs mit Gesundheit. Dazu wurden über 500 Personen online befragt. Die Ergebnisse bestätigen frühere Studien, dass die Nutzer von Wearables sich eher gesundheitsbewusst verhalten, also sportlich aktiver sind und auch stärker auf eine ausgewogene Ernährung achten als Personen, die keine Fitnesstracker verwenden. Die Nutzer gaben auch mehrheitlich an, dass sich ihr Gesundheitsverhalten deutlich verbessert hat, seitdem sie ein Wearable nutzen. Frauen waren häufiger von den positiven Effekten der Wearables überzeugt als Männer.

\section{Motivationshilfe}

Überraschenderweise verfügen Träger von Wearables - obwohl sie Daten und Informationen als Grundlage für ihr Gesundheitsverhalten nutzen - jedoch nicht generell über mehr Gesundheitswissen, zeigte die Studie. Nutzer kennen zwar häufiger ihren Blutdruck, aber Blutzuckerund Cholesterinwerte sind nur etwa jedem fünften Nutzer bekannt, und damit nicht häufiger als den Nicht-Nutzern. Besonders schlecht schneiden Personen ab, wenn sie nach Krankheitsrisiken befragt werden: Weniger als jeder sechste Befragte kennt die Hauptrisiken für Herz-Kreislauferkrankungen und weniger als jeder Zehnte kennt die Risiken für die Entstehung von Lungenkrebs - unabhängig davon, ob sie ein Wearable tragen oder nicht.

Quelle: Hochschule Fresenius: www.hs-fresenius.de 